\title{
Dual Sampling-Rate Observer Design for an Auto Operation Robot
}

\author{
C. Nakagome, H. Ogawa, H. Shibasaki, R. Tanaka, K., and Y. Ishida
}

\begin{abstract}
This paper describes dual sampling-late observer design for an auto operation robot. The proposed method has two sampling times to make the system more stability. For the plant, one depends on its sensor resolution. The other depends on the system. The former is longer than the latter. In this study, we could equalize the former to the latter with dual sampling-rate observer. In addition, the proposed method, which is used to realize the design, is discretized by a zero-order-hold, and works as a one-order-hold to increase the stability and simplify the design. In this study, we simulated three different sampling terms. Simulation results revealed the effectiveness of our proposed method.
\end{abstract}

Index Terms - Dual sampling-rate observer, optimal control, one-order-hold, robot.

\section{INTRODUCTION}

In digital control systems, there is a target value $r(t)$, an output response $y(t)$, and a control input $u(t)$. These values are represented by the three terms $T_{r}, T_{y}$, and $T_{u}$, respectively. The control input term $T_{u}$ is usually determined by an actuator, the speed of a digital-to-analog (D/A) converter, or the CPU processing speed. The output response term $T_{y}$ is determined by a sensor or the speed of an analog-to-digital (A/D) converter. An actual control system is often limited by $T_{u}, T_{y}$, or both $T_{u}$ and $T_{y}$. Therefore, conventional digital control systems are based on $T_{u}$ and $T_{y}$, whichever is longer, such that it equals the three different terms. To resolve this issue, H. Fujimoto designed a general framework of multi-rate sampling control and adapted it to motion control systems [1]. In this study we introduce a multi-rate sampling control technique, in which the control input term $T_{u}$ is shorter than the output term $T_{y}$, because we assume that the output response term is relatively longer for hardware. Since we commonly assume that a D/A converter performs faster calculations than an $\mathrm{A} / \mathrm{D}$ converter, we can consider that there are many control systems which have $T_{u}$ shorter than $T_{y}$. In particular, the issue is noticeable in robots of visual servo systems because the sampling time of a camera is very long [2]-[10]. In addition, optimal control is one of the simple designs in modern control systems. When the sampling term is short, the optimal control is good, but when it is long, the performance is bad. The realization of good performance with long sampling times has been reported by the analysis of optimal control [11].

In this study, we designed a control system that obtains a

Manuscript received November 21, 2012; revised January 22, 2013.

The authors are with Graduate School of Science and Technology, Meiji University, Kawasaki, Kanagawa 214- 8571 Japan (e-mail:ce11064@meiji.ac.jp). stable output, regardless of the sampling term, by combining these techniques with a dual sampling-rate observer [12]-[13]. We commonly use A/D conversion in discrete-time systems. It is desirable that the plant is discretized by a one-order-hold; however, the design of a one-order-hold is difficult. Therefore, we designed a simple one-order-hold by applying a zero-order-hold and included it in the control system. In the following sections, we simulate our proposed method by using the transfer function of DC motor, because it is difficult to apply the robot of a visual servo system.

\section{THEORY}

\section{A. Optimal Control}

State space equations for a controllable and an observable controlled plant are expressed in (1):

$$
\begin{aligned}
& \dot{\boldsymbol{x}}(t)=\boldsymbol{A} \boldsymbol{x}(t)+\boldsymbol{b} u(t) \\
& y(t)=\boldsymbol{c x}(t)
\end{aligned}
$$

We define $r(t)$ as a reference value. Then, the error between the reference value and output is expressed in (2):

$$
e(t)=r(t)-y(t)
$$

Next, we differentiate (2) as follows:

$$
\dot{e}(t)=\dot{r}(t)-\dot{y}(t)
$$

We define $\dot{r}(t)=0$. Then, (3) becomes (4):

$$
\dot{e}(t)=\boldsymbol{c} \dot{\boldsymbol{x}}(t)
$$

Also, we differentiate (1).

$$
\ddot{\boldsymbol{x}}(t)=\boldsymbol{A} \dot{\boldsymbol{x}}(t)+\boldsymbol{b} \dot{u}(t)
$$

State space equations in (6) are obtained from (4) and (5):

$$
\begin{aligned}
& {\left[\begin{array}{l}
\ddot{\boldsymbol{x}}(t) \\
\dot{e}(t)
\end{array}\right]=\left[\begin{array}{ll}
\boldsymbol{A} & \mathbf{0} \\
\boldsymbol{c} & 0
\end{array}\right]\left[\begin{array}{l}
\dot{\boldsymbol{x}}(t) \\
e(t)
\end{array}\right]+\left[\begin{array}{l}
\boldsymbol{b} \\
0
\end{array}\right] \dot{u}(t)} \\
& e(t)=\left[\begin{array}{ll}
\mathbf{0} & 1
\end{array}\right]\left[\begin{array}{l}
\dot{\boldsymbol{x}}(t) \\
e(t)
\end{array}\right]
\end{aligned}
$$

We discretize the equation (6) using the sampling time $T_{u}$.

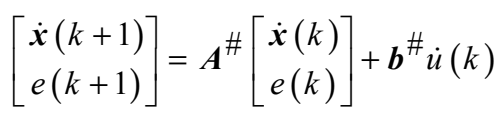

$$
\begin{aligned}
& e(k)=c^{\#\left[\begin{array}{c}
\dot{\boldsymbol{x}}(k) \\
e(k)
\end{array}\right]}
\end{aligned}
$$


Then, we express the equation to minimize the cost function as follows:

$$
J=\sum_{k=0}^{\infty}\left(\|e\|^{2} Q+\|\dot{u}\|^{2} R\right) .
$$

From (7) and (8), the control law $\dot{u}(k)$ is given by

$$
\dot{u}(k)=\boldsymbol{F}_{1} \dot{\boldsymbol{x}}(k)+f_{2} e(k) .
$$

The gains $F_{1}$ and $f_{2}$ are designed based on the LQI (Linear Quadratic Integral) meth

\section{B. Multi-Rate Sampling Method}

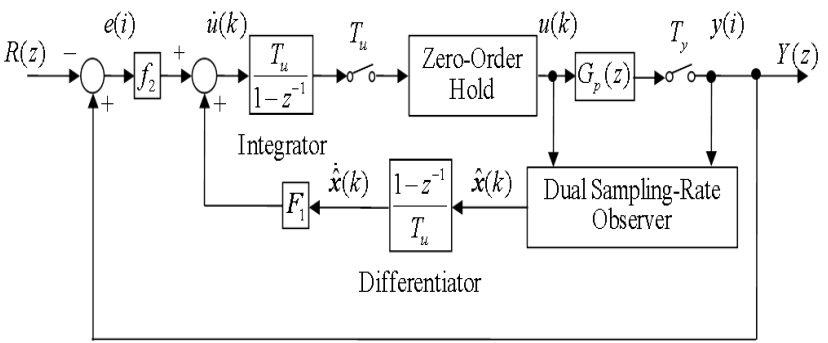

Fig. 1. The block diagram of the proposed method

We obtain the input based on an integrator, the input at the sampling time $k+1$ is given by

$$
u(k+1)=u(k)+\dot{u}(k)
$$

In this paper, multi-late sampling technique [1] is adopted. This technique is shown in Fig. 2.

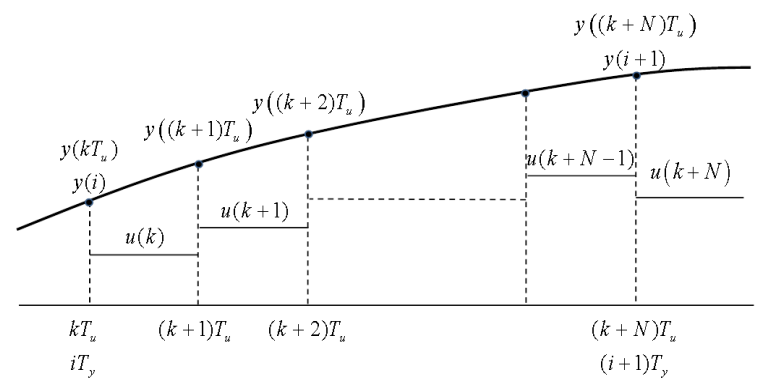

Fig. 2. Multi-rate system

In particular, the system output is observed once every $N=T_{y} / T_{u}$, where $N$ is an integer. Then, $T_{y}$ is the sampling time in the plant output and $T_{y}>T_{u}$. The sampling time $k$ in the system is defined by

$$
k=N i+j
$$

where $i=0,1, \cdots$ and $j=0,1, \cdots, N-1$

Therefore, (10) can be rewritten as

$$
\begin{aligned}
u(k+1) & =u(N i+j+1) \\
& =u(N i+j)+\dot{u}(N i+j)
\end{aligned}
$$

Similarly, the output equation is given by

$$
y(i)=c_{d} \boldsymbol{x}(N i),
$$

where $\boldsymbol{c}_{d}$ is obtained by discretizing the state space equations of the plant.

\section{Dual Sampling-Rate Observer}

We discretize the equation (1) with the sampling time $T_{y}$.

$$
\left.\begin{array}{l}
\boldsymbol{x}(k+1)=\boldsymbol{A}_{d} \boldsymbol{x}(k)+\boldsymbol{b}_{d} u(k) \\
y(k)=\boldsymbol{c}_{d} \boldsymbol{x}(k)
\end{array}\right\}
$$

Then, we express the dual sampling-late observer as follows:

$$
\left.\begin{array}{l}
\dot{\boldsymbol{x}}(k+1)= \\
\left\{\begin{array}{c}
A_{d} \dot{\boldsymbol{x}}(k)+\boldsymbol{b}_{d} u(k)+\boldsymbol{L}[y(k)-\dot{y}(k)], \text { for } k=N i \\
A_{d} \dot{\boldsymbol{x}}(k)+\boldsymbol{b}_{d} u(k), \text { otherwise }
\end{array}\right. \\
\dot{y}(k)=\boldsymbol{c}_{d} \dot{\boldsymbol{x}}(k)
\end{array}\right\}
$$

The equation (16) substituting (11) into (15):

$$
\begin{aligned}
& \dot{\boldsymbol{x}}(N i+j+1)= \\
& \left\{\begin{array}{l}
A_{d} \dot{\boldsymbol{x}}(N i+j)+\boldsymbol{b}_{d} u(N i+j) \\
+\boldsymbol{L}[y(N i+j)-\dot{y}(N i+j)], \text { for } j=0 \\
A_{d} \dot{\boldsymbol{x}}(N i+j)+\boldsymbol{b}_{d} u(N i+j), \text { otherwise }
\end{array}\right. \\
& \dot{y}(N i+j)=\boldsymbol{c}_{d} \dot{\boldsymbol{x}}(N i+j)
\end{aligned}
$$

Then, equation (9) can be rewritten as

$$
\begin{aligned}
\dot{u}(k) & =\dot{u}(N i+j) \\
& =\boldsymbol{F}_{1} \dot{\boldsymbol{x}}(N i+j)+f_{2} e(N i+j)
\end{aligned}
$$

where

$$
\left.\begin{array}{rl}
e(N i+j) & =r(N i+j)-y(N i+j) \\
& \approx r(N i)-y(N i)
\end{array}\right\}, \text { for } j=0,1, \cdots, N-1
$$

\section{SimULATION}

In this section, we compared results from our proposed method with those of the conventional method by performing simulation studies. The conventional method used the optimal control of a servo system. Dual sampling-rate observers are typically used in various plants. In these simulations, we used a DC motor, which is a simple and stable plant, and its state space equations are given as follows:

$$
\begin{aligned}
& \dot{\boldsymbol{x}}(t)=\left[\begin{array}{cc}
0 & 1 \\
0 & -4.96 \times 10
\end{array}\right] \boldsymbol{x}(t)+\left[\begin{array}{c}
0 \\
7.04 \times 10^{2}
\end{array}\right] u(t) \\
& y(t)=\left[\begin{array}{ll}
1 & 0
\end{array}\right] \boldsymbol{x}(t)
\end{aligned}
$$

Enhanced state space equation in this system is given by the equation (20).

$$
\begin{aligned}
& {\left[\begin{array}{l}
\ddot{\boldsymbol{x}}(t) \\
\dot{e}(t)
\end{array}\right]=\left[\begin{array}{ll}
\boldsymbol{A} & \mathbf{0} \\
\boldsymbol{c} & 0
\end{array}\right]\left[\begin{array}{l}
\dot{\boldsymbol{x}}(t) \\
e(t)
\end{array}\right]+\left[\begin{array}{l}
\boldsymbol{b} \\
0
\end{array}\right] \dot{u}(t)} \\
& e(t)=\left[\begin{array}{ll}
\mathbf{0} & 1
\end{array}\right]\left[\begin{array}{c}
\dot{\boldsymbol{x}}(t) \\
e(t)
\end{array}\right]
\end{aligned}
$$

where,

$$
\boldsymbol{A}=\left[\begin{array}{cc}
0 & 1 \\
0 & -4.96 \times 10
\end{array}\right], \boldsymbol{b}=\left[\begin{array}{c}
0 \\
7.04 \times 10^{2}
\end{array}\right],
$$




$$
\boldsymbol{c}=\left[\begin{array}{ll}
1 & 0
\end{array}\right]
$$

We discretize the equation (20) using the sampling time $T_{u}$.

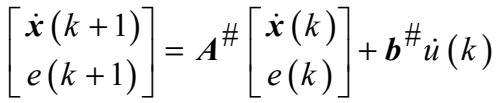

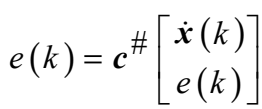

where,

$$
\begin{gathered}
\boldsymbol{A}^{\#}=\left[\begin{array}{ccc}
1 & 0.0156 & 0 \\
0 & 0.2258 & 0 \\
0.03 & 0.0003 & 1
\end{array}\right], \boldsymbol{B}^{\#}=\left[\begin{array}{l}
0.2043 \\
10.9883 \\
0.0023
\end{array}\right], \\
\boldsymbol{c}^{\#}=\left[\begin{array}{lll}
0 & 0 & 1
\end{array}\right] .
\end{gathered}
$$

In our simulation, we employ three sampling time conditions for this plant. A unit step signal was introduced as a target signal at $t=0[\mathrm{~s}]$. The weights of cost function $Q$ and $R$ are different in three cases, because it shows the same signal in the continuous time and the discrete time.

\section{A. Case 1}

In Case 1 , the control sampling time $T_{u}$ is $1.0[\mathrm{~ms}]$, and the output sampling time $T_{y}$ is $10[\mathrm{~ms}]$. The weights of the cost functions are $Q=1, R=1000$ in the conventional method (1[ms]), $Q=10, R=100$ in the conventional method (10[ms]), and $Q=1000, R=1$ in the proposed method. In addition, the observer gain is $L=\left[\begin{array}{lll}0.1664 & 12.2254\end{array}\right]^{\mathrm{T}}$.

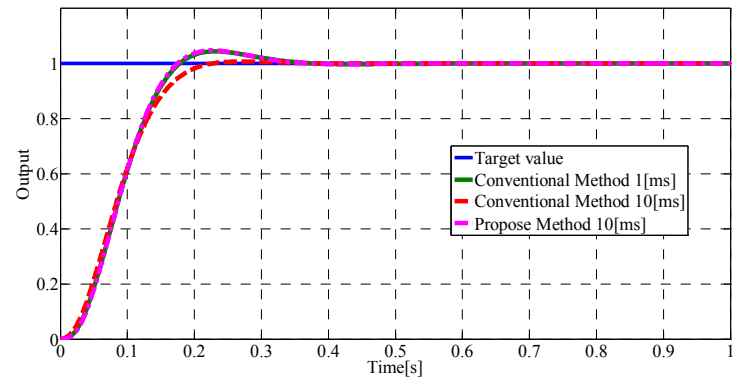

Fig. 3. Simulation result for CASE 1.

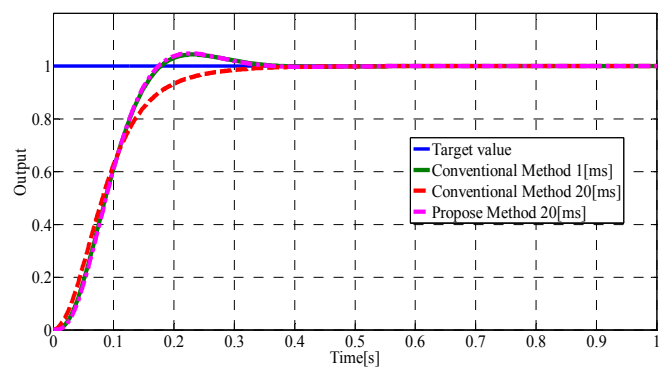

Fig. 4. Simulation result for Case 2.

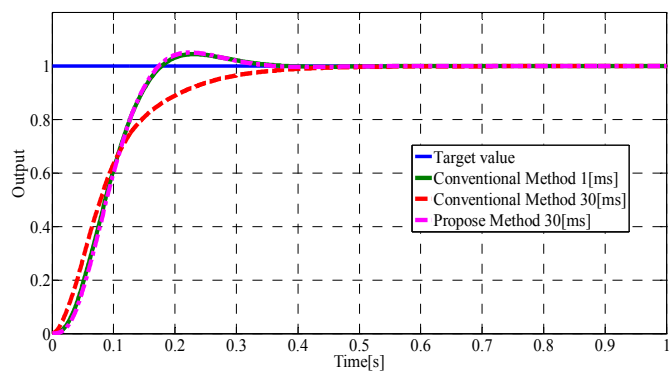

Fig. 5. Simulation result for Case 3.

\section{B. Case 2}

In Case 2, the control sampling time $T_{u}$ is $1.0[\mathrm{~ms}]$, and the output sampling time $T_{y}$ is $20[\mathrm{~ms}]$. The weights of the cost functions are $Q=1, R=1000$ in the conventional method (1[ms]), $Q=20, R=50$ in the conventional method (10[ms]), $Q=1000, R=1$ in the propose method. In addition, the observer gain is $L=[0.166412 .2254]^{\mathrm{T}}$.

\section{Case3}

In case 3 , the control sampling time $T_{u}$ is $1.0[\mathrm{~ms}]$, and the output sampling time $T_{y}$ is $30[\mathrm{~ms}]$. The weight of the cost function are $\mathrm{Q}=1, \mathrm{R}=1000$ in the conventional method (1[ms]), $Q=30, R=33$ in the conventional method ( $10[\mathrm{~ms}])$, $Q=1000, R=1$ in the propose method. And the observer gain is $L=\left[\begin{array}{lll}0.1664 & 12.2254\end{array}\right]^{\mathrm{T}}$.

\section{CONCLUSION}

In the paper, we have proposed a new control method for robots of a visual servo system. We have successfully designed a control method that realized a stable output by combining author's optimal control method [11], dual sampling-rate observer and multi-rate sampling technique. In the simulation studies, we have simulated the different three sampling terms. Simulation results have revealed that the proposed method has effectiveness. We would like to adapt our technique to the robot of visual servo system in the future work.

\section{REFERENCES}

[1] H. Fujimoto, "General Framework of Multirate Sampling Control and Applications to Motion Control Systems," PhD thesis, The University of Tokyo, 2000.

[2] K. Hashimoto and H. Kimura, "Visual servoing with nonlinear observer," IEEE Int. Conf. Robotics and Automation, pp. 484-489, 1995.

[3] H. Poor, An Introduction to Signal Detection and Estimation, New York: Springer-Verlag, ch. 4, 1985.

[4] M. Nemani, T. C. Tsao, and S. Hutchinson, "Multi-rate analysis and design of visual feedback digital servo-control system," ASME, J. Dynam. Syst., Measur, and Contr., vol. 116, pp. 44-55, 1994.

[5] L. E. Weiss, A. C. Sanderson, and C. P. Neuman, "Dynamic Sensor-Based Control of Robots with Visual Feedback," IEEE Journal of Robotics and Automation, vol. 3, no. 5, pp. 404-417, 1987.

[6] J. T. Feeddma and O. R. Mitchell, "Vision guided servoing with feature-based trajectory generation," IEEE Trans. Robotics and Automation, vol. 5, no. 5, pp. 691-700, 1989.

[7] H. Fujimoto, Y. Hori, T. Yamaguchi, and S. Nakagawa, "Proposal of perfect tracking and perfect disturbance rejection control by multirate sampling and applications to hard disk drive control," Conf. Decision Contr., pp. 5277-5282, 1999..

[8] H. Fujimoto and Y. Hori, "High performance servo systems based on multirate sampling control," Int. Fed. Autom. Control J. Control Eng. Practice, vol. 10, no. 7, pp. 773-781, 2002.

[9] H. Fujimoto and Y. Hori, "Visual servoing based on multirate sampling control," IEEE Int. Conf. Robotics and Automation, pp. 711-716, 2001.

[10] G. W. W. Chiang, "Multirate state-space digital controller for sector servo systems," Conf. Decision Contr., pp. 1902-1907, 1990.

[11] Y. Ishida and K. Furuta, "An application of Mechatronics," Computrol, no. 15 , pp. 87-93, Japanese.

[12] L. Kovudhikulrungsri and T. Koseki, "Precise Speed Estimation from a Low-Resolution Encoder by Dual-Sampling-Rate Observer," IEEE/ASME Trans. On Mechatronics, vol. 11, no. 6, pp. 661-670, 2006.

[13] T. Hara, Y. Takada, T. Koseki, and Y. Nozaki, Dual sampling rate digital signal processing for low speed vehicle tests, 2010. 


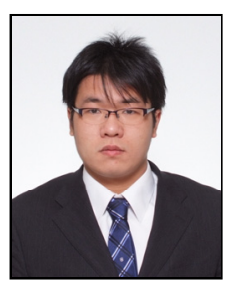

C. Nakagome was born in Nov. 1988 in Tokyo, Japan. He received the B. E. degree in Electronics and Bioinformatics from Meiji University, Kawasaki, Japan, in 2011. He is currently studying toward the M. E. degree at Graduate School of Science and Technology, Meiji University. His research interests include motion control of the robot car with labview.

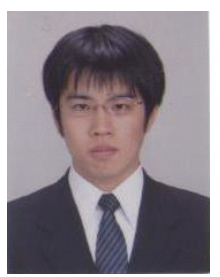

H. Shibasaki was born in Nov. 1988 in Tokyo, Japan. $\mathrm{He}$ received the B. E. degree in Electronics and Bioinformatics from Meiji University, Kawasaki, Japan in 2011. He is currently studying toward the M. E. degree at Graduate School of Science and Technology, Meiji University.

His research interests include continuous and digital control for a plant with integrator, and an unstable plant and its application to pneumatic equipment. $\mathrm{He}$ is a student member of the IEEE, and the IEEJ of Japan.

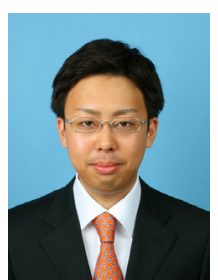

H. Ogawa was born in Tokyo, Japan, on August 4, 1984. He received the B. E. degree in Materials Science from Toyohashi University of Technology, Toyohashi, Japan, in 2007. He is currently working toward the M. E. degree at Graduate School of Electrical Engineering, Meiji University. He is currently studying toward the Dr. Eng. degree at Graduate School of Science and Technology, Meiji University. His research interests include digital signal processing and its applications. He is a member of the IEICE and SICE of Japan.

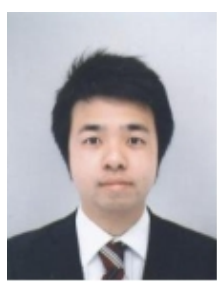

R. Tanaka was born in Oct. 1987 in Fukuoka, Japan He received the B. E. degree in Electronics and Communications from Meiji University, Kawasaki, Japan, in 2010. He received the M. E. degree in Graduate School of Science and Technology, Meiji University, in 2012.

$\mathrm{He}$ is currently studying toward the Dr. Eng. degree at Graduate School of Science and Technology, Meiji University. His research interests include Speech Signal Processing, Noise Reduction, and digital Control Systems.

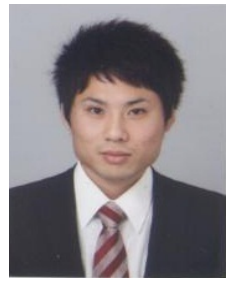

K. Kawaguchi was born in Feb. 1990 in Wakayama, JAPAN. He received the B. E. degree in Electronics and Bioinformatics from Meiji University, Kawasaki, Japan, in 2011. He is currently studying toward the M. E. degree at Graduate School of Science and Technology, Meiji University. His research interests include Dahlin Algorithm for a plant with time delay and its application to pneumatic equipment.

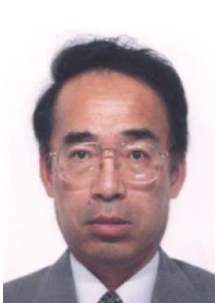

Y. Ishida was born in Tokyo, Japan. He received the B. E., M. E., and Dr. Eng. Degrees in Electrical Engineering, Meiji University, Kawasaki, Japan, in 1970, 1972, and 1978, respectively. In 1975 he joined the Department of Electrical Engineering, Meiji University, as a research Assistant and became a Lecturer and an Associate Professor in 1978 and 1981, respectively. He is currently a Professor at the Department of Electronics and Bioinformatics, Meiji University. His current research interests include signal processing, speech analysis and recognition, and digital control. He is a member of the IEEE, and the IEICE of Japan. 